\title{
Primordial Black Hole Dark Matter and Ways to Find It
}

\section{Volodymyr Takhistov*}

Department of Physics and Astronomy, University of California, Los Angeles

Los Angeles, California, 90095-1547, USA

Kavli Institute for the Physics and Mathematics of the Universe (WPI), UTIAS

The University of Tokyo, Kashiwa, Chiba 277-8583, Japan

E-mail: volodymyr.takhistov@ipmu.jp

Primordial black holes (PBHs) constitute an attractive non-particle dark matter (DM) candidate. We show how generic PBH formation models based on scalar fields can lead to PBHs with distinct features, including high spin as well as extended mass-spectrum compatible with multiple observations. We discuss how compact stars can serve as unique laboratories for exploring asteroid-mass PBHs constituting DM and also present new ways to test the origin of solar-mass and intermediate-mass PBHs.

40th International Conference on High Energy physics - ICHEP2020

July 28 - August 6, 2020

Prague, Czech Republic (virtual meeting)

\footnotetext{
*Speaker.
} 


\section{Introduction}

Conventional astrophysical black holes are formed at the end of stellar evolution from gravitational collapse. Black holes could also be primordial (PBHs), appearing in the early Universe [1-3], and constitute part or all of the dark matter (DM) (see [4-6] for review). PBHs have been associated with the recently observed gravitational wave (GW) signals (e.g. [7-10]), among others, and suggested as a key ingredient in resolution of major astrophysical puzzles.

PBHs are more ubiquitous than once thought and can appear in a variety of theoretical models [4-6]. In typical scenarios, PBHs form in the early Universe when an $\delta \sim O(1)$ overdensity perturbation enters the horizon after the end of inflation and subsequently collapses ${ }^{1}$. Since standard inflationary perturbations are nearly scale-invariant and must agree with CMB observations on cosmological scales, significant enhancements on small scales relevant for PBHs demand a significant degree of inflaton potential fine-tuning (e.g. [11, 12]). We discuss how new PBH formation mechanism from scalar field fragmentation avoids this problem and naturally leads to PBHs with large spin. Subsequently, we present another general PBH formation mechanism with scalars, based on vacuum bubble multiverse. This mechanism naturally leads to PBHs with a broad mass-spectrum that can simultaneously account for all DM as well as observations from Subaru HSC and LIGO.

New signatures and tests are needed to gain further insights into the role PBHs play in DM. We show that compact stars constitute attractive laboratories for exploring asteroid-mass PBHs that can constitute all of DM. Such interactions lead to a variety of signatures as well as pathways for potential resolution of long-standing astronomical puzzles. We discuss new ways to test PBHs in the solar and intermediate mass-ranges using BH mass-distribution and gas heating, respectively.

\section{PBHs from Scalar Fields}

Scalar fields generically appear in theories beyond the Standard Model (e.g. extra dimensions, string theories [13]). Models based on supersymmetry (SUSY) often include numerous scalars with vanishing potential and global $U(1)$ charge [14]. In the early Universe after inflation such fields coherently oscillate around the minimum of the potential and in the presence of attractive self-interactions (potential shallower than quadratic) can develop instabilities ${ }^{2}$ and fragment into solitonic lumps, Q-balls (complex field) [15] or oscillons (real field) (e.g. [16]). The field can either be spectator or the inflaton [17]. The lumps can be effectively long-lived ${ }^{3}$ and from numerical simulations [18-20] are expected to be large (percent of the horizon size at fragmentation). Scalar fragments allow for an onset of matter-dominated era after inflation.

The fragmentation process is stochastic and rare sub-horizon scalar lump overdensities could be sufficiently large to collapse into PBHs [21-24]. In Fig. 1 we display the resulting PBH massspectrum for several models (A, B, C), along with existing constraints. Intriguingly, scalar fields associated with electroweak-scale supersymmetry can naturally result in the PBH masses for which there are no observational constraints and that can account for all DM [24]. In this formation mechanism perturbations leading to PBHs are not related to inflation. PBHs to can form either before or after reheating and can originate either from inflaton or an unrelated spectator field.

${ }^{1} \mathrm{PBHs}$ can in principle appear within the standard cosmology, although the probability of this is extremely suppressed. ${ }^{2}$ This is analogous to gravitational Jeans instability.

${ }^{3}$ For Q-balls stability stems from charge conservation, although it is affected by higher dimensional operators. 

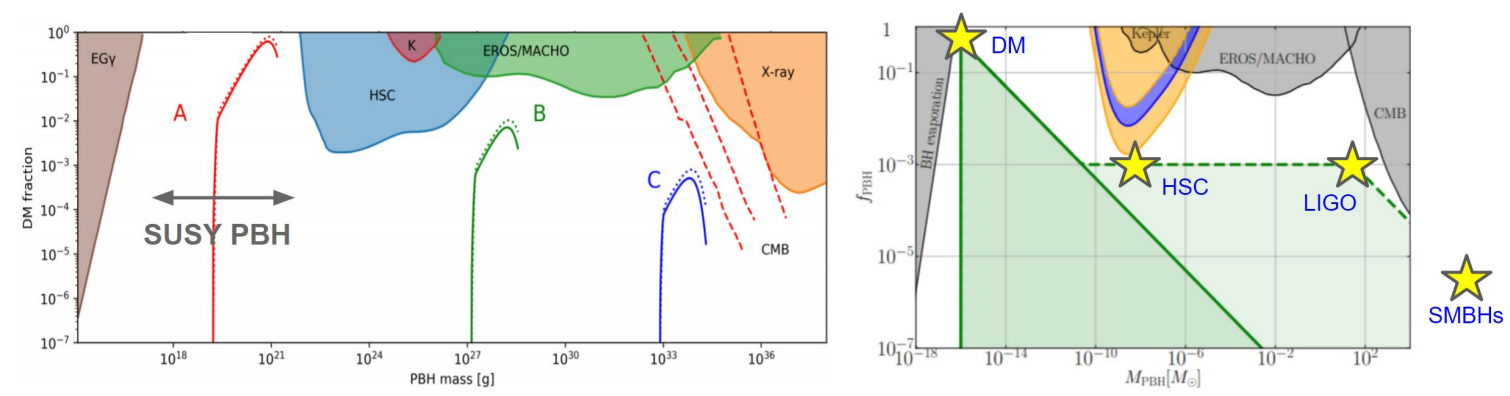

Figure 1: [Left] Models (A,B,C) of PBHs formed from scalar fragmentation, along with existing constraints, from [24]. Mass range of PBHs associated with electro-weak scale supersymmetry is shown. [Right] PBHs from generalized model of vacuum bubble multiverse, consistent with observations of DM, detected HSC and LIGO events as well as seeds of supersmassive BHs, from [28]. Yellow region denotes the reach of HSC observations required to definitely test this formation mechanism as the dominant source of PBH DM.

Importantly, $\mathrm{PBH}$ formation occurs during matter-dominated era and resulting $\mathrm{PBHs}$ can possess significant spin.

Another general PBH formation mechanism associated with scalars is that of vacuum bubble multiverse [25-27]. In models of multi-field inflation, the vacuum structure will generically be complicated. During inflation, the slow-rolling inflaton can quantum tunnel to near-by minima, resulting in vacuum bubble nucleation. As the bubbles expand, some are of such size (super-critical) that they are able to support inflation and a complicated space-time multiverse structure within. Subsequently, bubbles collapse to BHs. A distribution of bubbles in size naturally leads to PBHs with an extended mass-spectrum.

As demonstrated in [28], mass-spectrum of PBHs from vacuum bubble multiverse constituting all of DM is consistent with the recently reported microlensing event by Subaru HSC [29]. Upcoming HSC observations will definitely test if this production mechanism is the dominant source of PBH DM. Interestingly, as displayed on Fig. 1, a broad multi-step PBH mass-spectrum arising from generalization of this mechanism allows to simultaneously explain all of DM, observations from HSC and LIGO as well as seeds of supermassive BHs. HSC can also definitely test [30] extended mass-spectrum of PBH DM arising from models based on flat power spectrum that can simultaneously explain [31] the reported GW signal from NANOGrav [32].

\section{Compact Stars as PBH Laboratories}

PBH interactions with compact stars (neutron stars - NS, white dwarfs - WD) can result in a variety of distinct signatures. Detection of kilonova and electromagnetic emission from GW170817 NS-NS merger event [33] has been taken as evidence that this is the main source of r-process nucleosynthesis heavy element material (e.g. gold) abundance. However, the efficacy of merger contributions has been recently challenged [34]. As found in [35], potentially significant amount of r-process material can be produced from small PBHs that constitute DM being captured [36] and consuming NSs in DM rich environments such as Galactic Center. More so, such events can simultaneously account for heavy element abundance in the Milky Way as well as ultra-faint dwarf spheroidal galaxies, which show evidence of significant heavy element abundance consistent with a rare historic event [37]. Interestingly, compact stars interacting with PBHs are also potential sources of a variety of other 


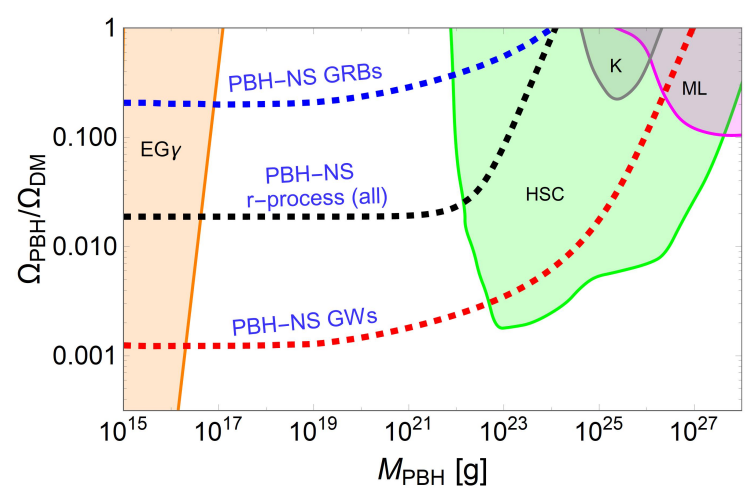

Figure 2: Signals from PBH-compact star interactions along with experimental constrains. Allowed parameter space (blue dashed) for PBH-NS gamma-ray bursts to significantly contribute to the observed positron excess at high energies [39]. Allowed parameter space (black dashed) where heavy element nucleosynthesis associated with PBH-NS systems can account for all of $r$-process element abundance in Milky Way as well as ultra-faint dwarf spheroidal galaxies simultaneously [35]. Allowed parameter space (red dashed) where GWs from transmuted binaries from PBH-NS interactions can be within observation reach of LIGO, assuming a lower signal-to-noise threshold than design [38]. Dashed lines correspond to the maximum reach for each signal, assuming the most optimistic input parameter choice for the fits.

signatures [35, 38, 39], including positrons and the associated $511 \mathrm{keV}$ radiation - relevant for signal observed from the Galactic Center (see also [40]), fast radio bursts, "orphan kilonovae" (without accompanying GWs), "transmuted" solar mass BHs left after small captured PBHs consume the NSs as well as microquasars and "orphan gamma-ray bursts" (without accompanying GWs). We display the PBH parameter space associated with some of these signals in Fig. 2.

\section{New Tests for Solar-mass and Intermediate-mass PBHs}

It is important to devise tests to probe PBHs over a broad mass range and also to distinguish them from other BHs. BHs below $\sim 2.5 M_{\odot}$ are not expected from conventional stellar evolution. Recently, LIGO has report $\sim 1.5-2.6 M_{\odot} \mathrm{BH}$ candidate event [41]. A simple yet powerful approach to test if such BHs are transmuted NSs due to DM/PBH interactions or PBHs is to examine if their mass-distribution tracks that of NSs [42], as displayed on Fig. 3. This demonstrates that events with $\mathrm{BH}$ mass $>1.5 M_{\odot}$ are unlikely to be transmuted.

LIGO has also recently reported first detection of intermediate-mass $\mathrm{BH}$ merger event with a total mass of $150 M_{\odot}$ [43]. While a multitude of constraints exist in this PBH mass-range, they often rely on a variety of assumptions. A new cosmology-independent test for intermediate-mass $\mathrm{BHs}$ has been proposed in [44] based on PBHs interacting and heating surrounding interstellar medium gas. Using DM-rich Leo T galaxy as target and taking into account heating processes due to dynamical friction, emission from BH accretion disk and outflows/winds, PBHs are constrained over $\sim 10-10^{7} M_{\odot}$ mass-range as displayed on Fig. 3. Gas heating can also be used to test small evaporating PBHs [45, 46]. 

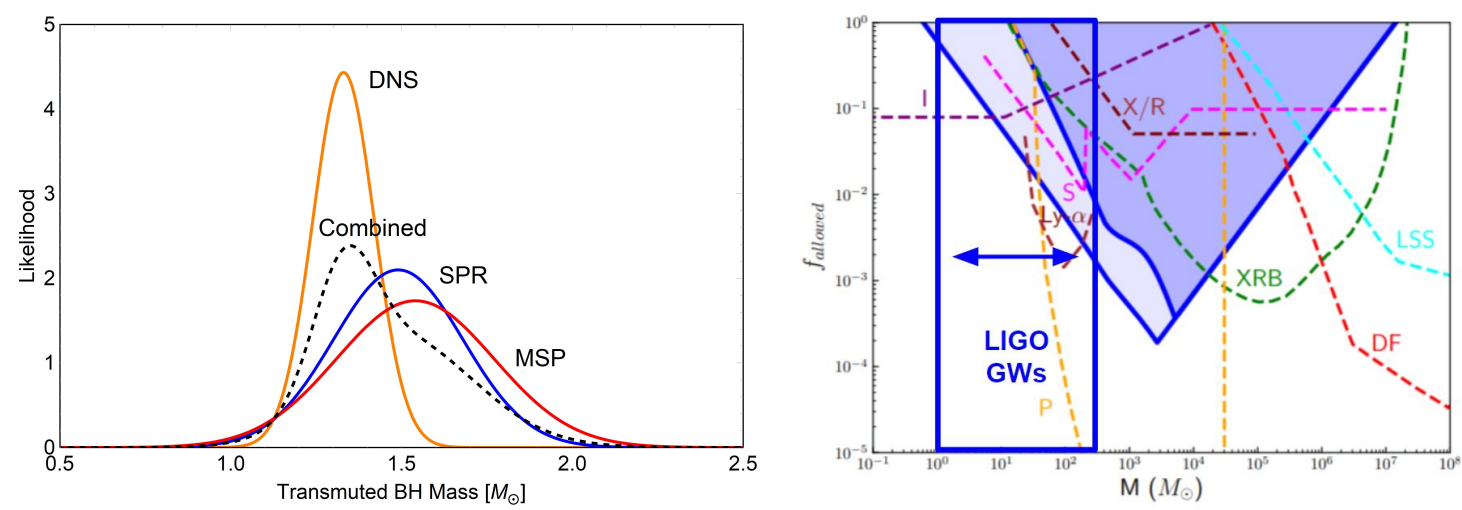

Figure 3: [Left] Expected mass distribution of "transmuted" solar-mass BHs, from [42]. [Right] Constraints on PBHs from gas heating of Leo T galaxy, from [44].

\section{Summary}

Intimate connection exists between PBHs and GW/multi-messenger astronomy. As we have shown, PBHs can be produced in generic models with scalar fields with a variety of properties, including high spin and broad mass-distribution. Compact stars can act as laboratories for asteroid-mass PBHs that constitute all of DM and are yet unconstrained, allowing for variety of novel signals and potential opportunities to alleviate existing astronomy puzzles. PBHs in the solar-mass range as well as intermediate mass-range can be tested via mass-distribution as well as gas heating, respectively. This could shed light on the origin of the recently detected GW signals.

\section{Acknowledgements}

We thank the organizers for the opportunity to present these results. This work of was supported by the U.S. Department of Energy (DOE) Grant No. DE-SC0009937 and by the World Premier International Research Center Initiative (WPI), MEXT, Japan.

\section{References}

[1] Y. B. Zel'dovich and I. D. Novikov, Sov. Astron. 10 (1967) 602.

[2] S. Hawking, Mon. Not. Roy. Astron. Soc. 152 (1971) 75.

[3] B. J. Carr and S. W. Hawking, Mon. Not. Roy. Astron. Soc. 168 (1974) 399.

[4] B. Carr, K. Kohri, Y. Sendouda and J. Yokoyama, 2002 . 12778.

[5] M. Sasaki, T. Suyama, T. Tanaka and S. Yokoyama, Class. Quant. Grav. 35 (2018) 063001 [1801.05235].

[6] M. Yu. Khlopov, Res. Astron. Astrophys. 10 (2010) 495 [0801. 0116].

[7] T. Nakamura, M. Sasaki, T. Tanaka and K. S. Thorne, Astrophys. J. 487 (1997) L139 [astro-ph/9708060].

[8] S. Clesse and J. Garcia-Bellido, Phys. Rev. D92 (2015) 023524 [1501.07565].

[9] S. Bird et al., Phys. Rev. Lett. 116 (2016) 201301 [1603.00464].

[10] M. Sasaki, T. Suyama, T. Tanaka and S. Yokoyama, Phys. Rev. Lett. 117 (2016) 061101 [1603.08338].

[11] M. Kawasaki and V. Takhistov, Phys. Rev. D98 (2018) 123514 [1810.02547]. 
[12] C. Germani and I. Musco, Phys. Rev. Lett. 122 (2019) 141302 [1805. 04087].

[13] A. Arvanitaki, S. Dimopoulos, S. Dubovsky, N. Kaloper and J. March-Russell, Phys. Rev. D81 (2010) 123530 [0905.4720].

[14] T. Gherghetta, C. F. Kolda and S. P. Martin, Nucl. Phys. B468 (1996) 37 [hep-ph/9510370].

[15] S. R. Coleman, Nucl. Phys. B262 (1985) 263.

[16] S. Kasuya, M. Kawasaki and F. Takahashi, Phys. Lett. B559 (2003) 99 [hep-ph/0209358].

[17] M. A. Amin, 1006. 3075.

[18] A. Kusenko and M. E. Shaposhnikov, Phys. Lett. B418 (1998) 46 [hep-ph/9709492].

[19] S. Kasuya and M. Kawasaki, Phys. Rev. D62 (2000) 023512 [hep-ph/0002285].

[20] T. Multamaki and I. Vilja, Phys. Lett. B535 (2002) 170 [hep-ph/0203195].

[21] E. Cotner and A. Kusenko, Phys. Rev. Lett. 119 (2017) 031103 [1612. 02529].

[22] E. Cotner and A. Kusenko, Phys. Rev. D96 (2017) 103002 [1706.09003].

[23] E. Cotner, A. Kusenko and V. Takhistov, Phys. Rev. D98 (2018) 083513 [1801.03321].

[24] E. Cotner, A. Kusenko, M. Sasaki and V. Takhistov, JCAP 10 (2019) 077 [1907 . 10613].

[25] J. Garriga, A. Vilenkin and J. Zhang, JCAP 02 (2016) 064 [1512 . 01819].

[26] H. Deng and A. Vilenkin, JCAP 12 (2017) 044 [1710.02865].

[27] H. Deng, A. Vilenkin and M. Yamada, JCAP 07 (2018) 059 [1804 . 10059].

[28] A. Kusenko, M. Sasaki, S. Sugiyama, M. Takada, V. Takhistov and E. Vitagliano, Phys. Rev. Lett. 125 (2020) 18 [2001.09160].

[29] H. Niikura et al., Nat. Astron. 3 (2019) 524 [1701. 02151].

[30] S. Sugiyama, V. Takhistov, E. Vitagliano, A. Kusenko, M. Sasaki and M. Takada, 2010.02189.

[31] V. De Luca, G. Franciolini and A. Riotto, 2009.08268.

[32] NANOGRAV collaboration, Z. Arzoumanian et al., 2009.04496.

[33] VIRgo, LIGO collaboration, B. P. Abbott et al., Phys. Rev. Lett. 119 (2017) 161101 [1710.05832].

[34] C. Kobayashi, A. I. Karakas and M. Lugaro, Astrophys. J. 900 (2020) 179 [2008 . 04660].

[35] G. M. Fuller, A. Kusenko and V. Takhistov, Phys. Rev. Lett. 119 (2017) 061101 [1704.01129].

[36] F. Capela, M. Pshirkov and P. Tinyakov, Phys. Rev. D87 (2013) 123524 [1301 .4984].

[37] A. P. Ji, A. Frebel, A. Chiti and J. D. Simon, 531 (2016) 610 [1512 . 01558].

[38] V. Takhistov, Phys. Lett. B782 (2018) 77 [1707.05849].

[39] V. Takhistov, Phys. Lett. B789 (2019) 538 [1710.09458].

[40] G. M. Fuller, A. Kusenko, D. Radice and V. Takhistov, Phys. Rev. Lett. 122 (2019) 121101 [1811.00133].

[41] LIGO Scientific, Virgo collaboration, R. Abbott et al., Astrophys. J. Lett. 896 (2020) L44 [2006.12611].

[42] V. Takhistov, G. M. Fuller and A. Kusenko, 2008.12780.

[43] LigO Scientific, Virgo collaboration, R. Abbott et al., Phys. Rev. Lett. 125 (2020) 101102 [2009.01075].

[44] P. Lu, V. Takhistov, G. B. Gelmini, K. Hayashi, Y. Inoue and A. Kusenko, 2007. 02213.

[45] H. Kim, 2007.07739.

[46] R. Laha, P. Lu and V. Takhistov, 2009.11837. 